sequencing can identify the existence or absence of tumor microenvironments permissive for checkpoint therapy. They provide a transcriptional signature that serves to delineate an "immunologically hot" from an "immunologically cold" tumor immune microenvironment (TiME). Through the rigor of their transcriptome-based molecular subtyping, followed by additional validation, Jang and colleagues ${ }^{1}$ have identified a set of markers that are associated with a favorable response to anti-PD-1/ P-L1 therapy for lung adenocarcinomas and squamous carcinomas. This well-performed study highlights an inherent advantage of messenger RNA sequencing, in which a particular gene's influence is amplified in the cell's transcriptome and thus is less susceptible to signal dropout than is sequencing single-copy genomes for variants. Although the interpretation of hundreds of genes is less tangible than a handful of driver mutations at the genome level, messenger RNA sequencing can provide signatures that, as a panel, are clinically useful and mimic the strength behind the use of polygenic risk scores from genome-wide studies.

The greater significance of the work by Jang and colleagues ${ }^{1}$ is that their granular approach for uncovering potential mechanisms of PD-1/PD-L1 resistance may facilitate an enhanced understanding of successful checkpoint inhibition. The "Good TiME" and "Bad TiME" gene signatures highlight active contributions from multiple cell types, and thus this is an early step in the process of better characterizing novel therapeutic potentials within the tumor microenvironment. The findings of active B-cell-derived gene activity outside T-cell-derived PD-1/PD-L1 pathways provides a translational foundation for a future of multimodal immunologic therapy impervious to single-agent resistance. The work of these joint investigators opens up the possibility that intervening at multiple derivative levels of checkpoint inhibition may be associated with enhancing the efficacy of anti-PD-1/ PD-L1 therapy, which would truly signal good times. In the end, the accumulation of more time to pursue more data on transcriptome-driven subtyping may actually inform all of us of better TiMEs to come.

\section{Reference}

1. Jang HJ, Lee HS, Ramos D, Park IK, Hang CH, Burt BM, et al. Transcriptomebased molecular subtyping of non-small cell lung cancer may predict response to immune checkpoint inhibitors. J Thorac Cardiovasc Surg. 2020;159: 1598-610.e3.

\title{
Commentary: The checkpoint before checkpoint inhibitors
}

\author{
William A. Blessing, PhD, and \\ Yolonda L. Colson, MD, PhD
}

Until recently, few therapeutic options existed for patients with unresectable non-small cell lung cancer (NSCLC) - particularly if targetable mutations within the tumor were not present, as is common in squamous cell lung cancer. Results from several recent clinical trials

\footnotetext{
From the Division of Thoracic Surgery, Harvard Medical School and Massachusetts General Hospital, Boston, Mass.

Disclosures: Authors have nothing to disclose with regard to commercial support.

Received for publication Nov 25, 2019; accepted for publication Nov 25, 2019; available ahead of print Feb 4, 2020.

Address for reprints: Yolonda L. Colson, MD, PhD, Division of Thoracic Surgery, Massachusetts General Hospital, 55 Fruit St, Boston, MA 02114 (E-mail: ycolson@mgh.harvard.edu).

J Thorac Cardiovasc Surg 2020;159:1614-5

$0022-5223 / \$ 36.00$

Copyright (c) 2019 by The American Association for Thoracic Surgery

https://doi.org/10.1016/j.jtcvs.2019.11.096
}

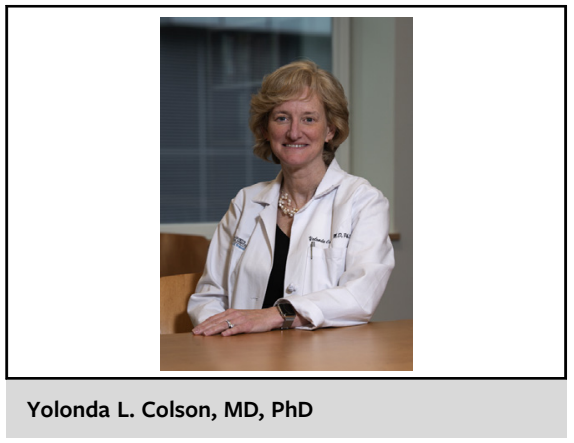

CENTRAL MESSAGE

Transcriptomic profiling of

NSCLC may aid in screening and identifying patients with immunologically active tumor microenvironments that could benefit from immunologic checkpoint inhibition. 
with immune checkpoint inhibitors have fundamentally altered the treatment of solid tumors, including NSCLC, as a result of the reversal of immune resistance orchestrated by the tumor microenvironment. However, the identification of biomarkers that can predict individual tumor response to these new immunotherapeutic agents is a critical need in this rapidly growing field, particularly given significant tumor heterogeneity even within the same histopathologic subset.

The study by Jang and colleagues ${ }^{1}$ in the current issue of the Journal of Thoracic and Cardiovascular Surgery extends "personalization" of cancer therapy to the development and validation of immunogenomic classifications of NSCLC by using transcriptomic data, with the ultimate goal of predicting tumor response to immune checkpoint inhibitors. The authors ${ }^{1}$ have used transcriptomic data obtained from surgically resected NSCLC specimens (87 adenocarcinoma and 101 squamous cell) and the universally available Barcelona cohort expression data sets to identify 59 genes in NSCLC tumors that serve as identifiers for tumor microenvironments susceptible to immunomodulation. There were 3 distinct immunomolecular subtypes in adenocarcinoma and 2 subtypes in squamous cell carcinoma identified by hierarchic clustering with characterization of "immunologically hot" tumors as exhibiting high cytolytic activity, immune cell infiltration, and immunoregulatory molecules, in addition to an interferon- $\gamma$ signaling signature. Retroactive application of these criteria to a separate, albeit small, cohort from the available Barcelona data set revealed a positive correlation between immunologically hot subtyping and favorable clinical responses to programmed death ligand
1 blockade. In contrast, this correlation was not seen with the "immunologically cold" immunomolecular subtypes, which were characterized by fewer immune regulatory molecules, MHC molecules, and CD8 T cells, but, interestingly, a higher fraction of CD4 regulatory $\mathrm{T}$ cells. This work reminds us that immunotherapy is not just about unleashing the inhibited $\mathrm{T}$ cell and further highlights the importance of the interplay between the patient's immune status and the heterogeneity of the tumor microenvironment in determining the response to checkpoint inhibitors.

The most interesting aspect of this study is thus not the placement of various lung carcinomas into transcriptomedefined bins according to their immunomolecular clustering, but rather the potential of creating a platform that is based on tumor microenvironmental immunomolecular subtypes and capable of preemptively assessing efficacy of immunotherapy. Although larger patient cohorts and prospective analyses are required to confirm these findings before direct clinical translation, the potential opportunity to create a therapeutic checkpoint whereby patients are individually selected for treatment (i.e., checkpoint inhibitors versus other systemic therapies) as a function of their immunomolecular profiles and likelihood of response is quite exciting and holds the promise of further shifting the treatment paradigm for NSCLC toward immunotherapy.

\section{Reference}

1. Jang HJ, Lee HS, Ramos D, Park IK, Hang CH, Burt BM, et al. Transcriptomebased molecular subtyping of non-small cell lung cancer may predict response to immune checkpoint inhibitors. J Thorac Cardiovasc Surg. 2020;159: 1598-610.e3. 